\title{
Analysis of CCR7 mediated T cell transfectant migration using a microfluidic gradient generator
}

\author{
Xun $\mathrm{Wu}^{\mathrm{a}}{ }^{\mathrm{a} b}$, Jiandong $\mathrm{Wu}^{\mathrm{a}, \mathrm{c}}$, Hongzhao Li ${ }^{\mathrm{b}}$, Daniel F. Legler ${ }^{\mathrm{d}}$, Aaron J. Marshall ${ }^{\mathrm{b}}$, Francis Lin ${ }^{\mathrm{a}, \mathrm{b}, \mathrm{c}, *}$ \\ a Department of Physics and Astronomy, University of Manitoba, Winnipeg, MB, R3T 2N2, Canada \\ ${ }^{\mathrm{b}}$ Department of Immunology, University of Manitoba, Winnipeg, MB, R3E OT5, Canada \\ c Department of Biosystems Engineering, University of Manitoba, Winnipeg, MB, R3T 2N2, Canada \\ d Biotechnology Institute Thurgau (BITg), University of Konstanz, Unterseestrasse 47, CH-8280 Kreuzlingen, Switzerland
}

Keywords:

Cell migration

Chemotaxis

T cells

Microfluidic device

CCR7

CCL19

\begin{abstract}
A B S T R A C T
T lymphocyte migration is crucial for adaptive immunity. Manipulation of signaling molecules controlling cell migration combined with in vitro cell migration analysis provides a powerful research approach. Microfluidic devices, which can precisely configure chemoattractant gradients and allow quantitative single cell analysis, have been increasingly applied to cell migration and chemotaxis studies. However, there are a very limited number of published studies involving microfluidic migration analysis of genetically manipulated immune cells. In this study, we describe a simple microfluidic method for quantitative analysis of $\mathrm{T}$ cells expressing transfected chemokine receptors and other cell migration signaling probes. Using this method, we demonstrated chemotaxis of Jurkat transfectants expressing wild type or C terminus mutated CCR7 within a gradient of chemokine CCL19, and characterized the difference in transfectant migration mediated by wild type and mutant CCR7. The EGFP tagged CCR7 allows identification of CCR7 expressing transfectants in cell migration analysis and microscopy assessment of CCR7 dynamics. Collectively, our study demonstrated the effective use of the microfluidic method for studying CCR7 mediated T cell transfectant migration. We envision this developed method will provide a useful platform to functionally test various signaling mechanisms at the cell migration level.
\end{abstract}

\section{Introduction}

Chemoattractant concentration gradient directed immune cell migration (chemotaxis) critically orchestrates the trafficking and homing of various immune cell types in tissues (Cahalan and Parker, 2008; Jin et al., 2008; Gambardella and Vermeren, 2013; Heuzé et al., 2013). T lymphocytes are the key players in adaptive immunity (Garside et al., 1998; Miller et al., 2002; Bromley et al., 2008; John et al., 2009). These cells have highly regulated tissue specific migratory properties (Campbell and Butcher, 2000; Moser and Loetscher, 2001; Kunkel and Butcher,

\footnotetext{
* Corresponding author at: Department of Physics and Astronomy, University of Manitoba, Winnipeg, MB R3T 2N2, Canada.

E-mail address: flin@physics.umanitoba.ca (F. Lin).
}

2002). In particular, chemokine receptor CCR7 is required for naïve and central memory $\mathrm{T}$ cell migration to lymph nodes (LN), which is important to initiate adaptive immune response and immune surveillance (Forster et al., 1999, 2008; Gunn et al., 1999). Chemokine CCL21, a ligand for CCR7, is highly expressed in LN and is responsible for T cell recruitment to LN (Ngo et al., 1998; Luther et al., 2002). Chemokine CCL19, another ligand for CCR7, is a potent chemoattractant for CCR7 expressing T cells in vitro. However, CCL19 is only produced in LN at low level and seems not to participate in T cell recruitment to LN (Luther et al., 2002; Link et al., 2007). CCL19 but not CCL21 triggers robust CCR7 desensitization and internalization (Bardi et al., 2001; Kohout et al., 2004). Interestingly, deletion of the $C$ terminus of CCR7 does not completely inhibit the CCL19 induced internal ization (Otero et al., 2008). The mechanisms underlying these unique features of CCR7 and their roles in mediating T cell 
migration are not clear. Further studies to better understand CCR7 mediated T cell migration will benefit from an advanced experimental platform, which is capable of quantitative single cell migration analysis and imaging studies of cell migration signaling at the molecular level in well defined chemical gradients.

Most in vitro studies of $\mathrm{T}$ cell migration employed the conventional transwell assay. Its capacity is limited by poor gradient control and inability to visualize migrating cells (Keenan and Folch, 2008). Microfluidic devices, which can precisely configure gradient conditions, offer useful tools for cell migration and chemotaxis studies (Li and Lin, 2011; Wu et al., 2013). Previously, we used a microfluidic gradient generating device to study the migration of activated human peripheral blood T cells (ahPBT) (Lin and Butcher, 2006; Nandagopal et al., 2011). Microfluidic devices were also used to study the migration of other subsets of human peripheral blood T cells such as memory T cells (Lin et al., 2008). However, there have been few reports for studying signaling mechanisms in chemotaxing immune cells by combining genetic manipu lations and controlled chemoattractant gradient profiles gen erated by microfluidic devices, especially in lymphocytes (Long et al., 2004; Sai et al., 2006; Cavnar et al., 2011). Among them, several studies employed microfluidic devices to study chemo tactic signaling for neutrophil chemotaxis using neutrophil like cell lines expressing transfected mutants of specific signaling molecules such as CXCR2 (Sai et al., 2006) and Hax1 (Cavnar et al., 2011). We previously employed a microfluidic gradient generator to quantitatively evaluate the role of tandem $\mathrm{PH}$ domain containing protein 2 (TAPP2) for regulating malignant B lymphocyte migration (Li et al., 2013). However, the effective use of microfluidic device for studying $T$ cell transfectants chemotaxis targeting specific signaling mechanisms has not been demonstrated.

The potential of advanced microfluidic platforms to study chemotaxis mechanisms in immune cells is strengthened by recent development of molecular probes and imaging technol ogies (Wang et al., 2008; Deng et al., 2011). Immunofluores cence staining against specific cell surface and intracellular markers is a well established technique (Fritschy and Härtig, 2001; Robinson et al., 2010). For example, this method can be used to visualize surface distribution and endocytosis of chemoattractant receptors as well as cytoskeleton in migrating cells at the time point of interest (Ballestrem et al., 1998; Otero et al., 2006). In addition, advanced methods were developed to visualize the dynamics of signaling events in migrating cells in real time (Servant et al., 2000; Ridley et al., 2003; Riedl et al., 2008; Arai et al., 2010). New F actin binding probes have become available for real time visualization of cytoskeleton dynamics in cell migration (Riedl et al., 2008). State of the art imaging modules such as confocal and total internal reflection fluorescence microscopy (TIRF) have been widely used in cell migration research to enable high resolution molecular imag ing (Blow, 2007). It will provide a powerful research approach for studying $\mathrm{T}$ cell migration and chemotaxis by integrating molecular imaging with microfluidic devices.

The main goal of the present study is to demonstrate the feasibility of combining a microfluidic gradient generator with molecular immunology methods to quantitatively analyzing the migration of genetically modified T cell transfectants. We wanted to determine whether chemotactic signaling constructs can be effectively transfected to $\mathrm{T}$ cells and mediate functional migration and chemotaxis of the $\mathrm{T}$ cell transfectants in microfluidic devices. Furthermore, we wanted to examine the function of both wild type (wt) CCR7 and C terminus mutated CCR7 in mediating $\mathrm{T}$ cell transfectant chemotaxis in the microfluidic system.

\section{Materials and methods}

\subsection{Plasmid construction}

Lifeact RFP plasmid was purchased from ibidi GmbH. The CCR7 WT and CCR7 WT EGFP plasmids were described previ ously (Otero et al., 2008). The CCR7 C terminus truncated sequence was generated by polymerase chain reaction (PCR) using primer design 5' ATA GAA TTC CGT CAT GGA CCT GGG GAA AC 3' (EcoRI) (restriction site underlined) and 5' TGC GGC CGC GCC CAG GTC CTT GAA GAG C 3' (NotI) (restriction site underlined) based on CCR7 truncation site. This PCR product was digested by EcoRI and NotI and ligated into pcDNA3 CCR7 WT EGFP vector to remove CCR7 WT. After transformation into competent Escherichia coli, clones contain ing the truncated CCR7 insert were sequence verified.

\subsection{Cell line and transfection}

Jurkat $\mathrm{T}$ cells (human leukemia $\mathrm{T}$ cell) were cultured in RPMI 1640 medium containing 10\% fetal calf serum (FCS) and $1 \%$ penicillin streptomycin $(\mathrm{P} / \mathrm{S})$. Transient transfections were carried out by the Neon Transfection System following the manufacturer's protocol. Cells were passaged one day before transfection. $2.5 \times 10^{5}$ cells were resuspended in the $\mathrm{R}$ buffer containing $1 \mu \mathrm{g}$ plasmid in a $10 \mu \mathrm{L}$ Neon tip for each electro poration. The cells were electroporated at $1325 \mathrm{~V}$ pulse voltage, 10 ms pulse width for 3 pulses.

\subsection{Cell surface receptor expression}

CCR7 transfected Jurkat cells were stained with anti CCR7 Alexa647 (BD Biosciences) for $30 \mathrm{~min}$ on ice. After washing twice, cells were analyzed by flow cytometry using a flow cytometer (FACS Canto II, BD Biosciences). The flow data were further analyzed using Flowjo (Tree Star, OR).

\subsection{Transwell assay}

Transwell assays (Corning Inc, NY) were performed across bare polycarbonate membranes. Briefly, $5 \times 10^{5}$ cells in $100 \mu \mathrm{L}$ of medium were added to the top well of a $6.5 \mathrm{~mm}$ diameter transwell culture insert with a pore size of $5 \mu \mathrm{m}$. The insert was then transferred to wells containing $600 \mu \mathrm{L}$ of medium with or without different concentrations of chemoattractants. Cells were allowed to migrate in the transwell assay for $4 \mathrm{~h}$ at $37^{\circ} \mathrm{C}$ with $5 \% \mathrm{CO}_{2}$. Transmigrated cells into the lower well were resuspended in medium and collected for counting using a flow cytometer (FACS Canto II, BD Biosciences). The migration rate was measured as the percentage of the total input cells that migrated into the lower well. The flow data were further analyzed using Flowjo. At least triplicate was performed for the medium control group and CCL19 group. 


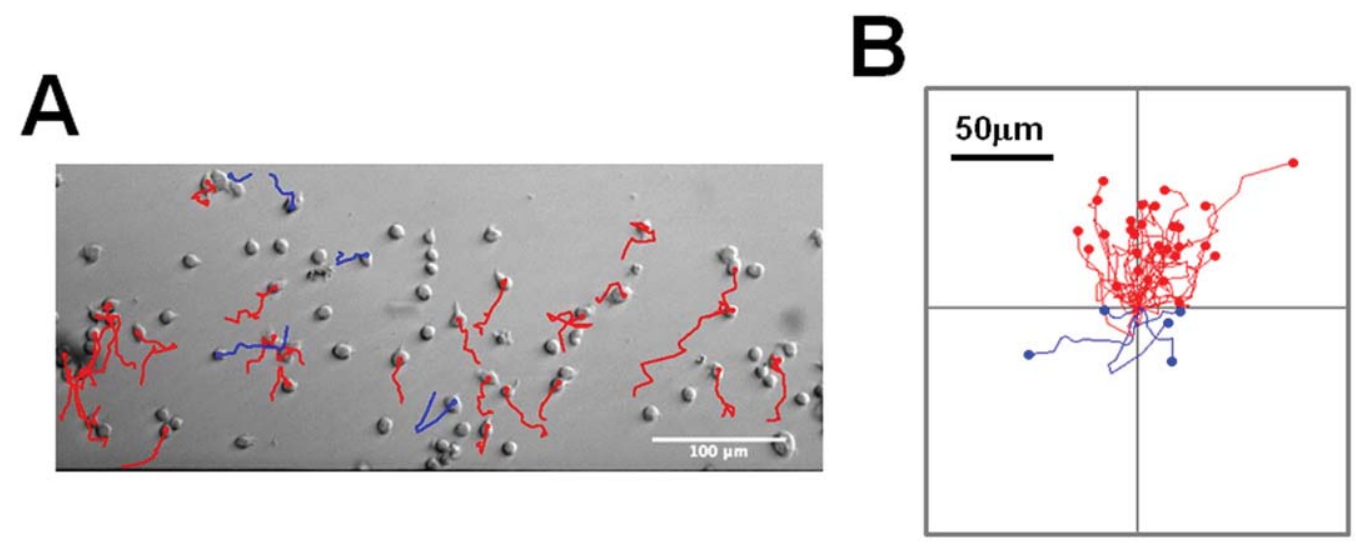

C
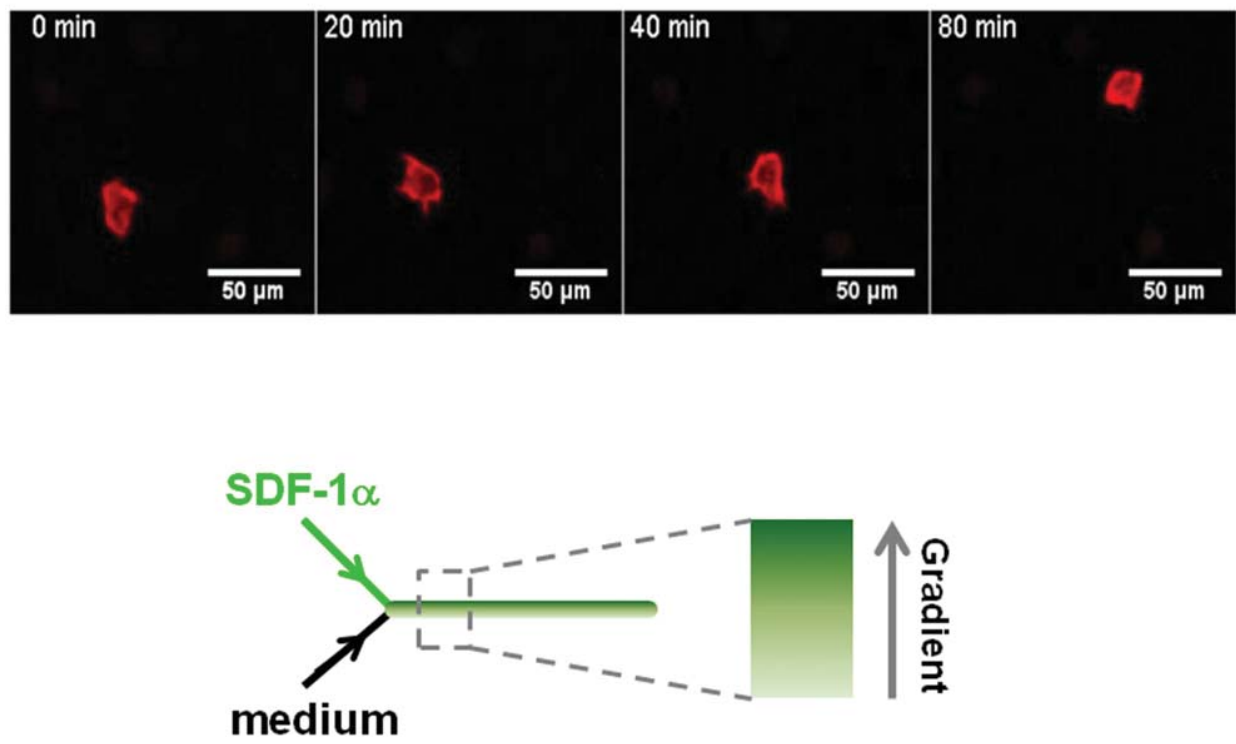

Fig. 1. Chemotaxis of parental Jurkat cells or Jurkat transfectant expressing Lifeact-RFP to a SDF-1 $\alpha$ gradient using the microfluidic platform. A) Cell movement paths were tracked for parental Jurkat cells migrating under a $100 \mathrm{nM} \mathrm{SDF}-1 \alpha$ gradient with higher SDF-1 $\alpha$ concentration at the top (red: toward the gradient; blue: away from the gradient). B) Plot of cell tracks in A) normalized to a common origin (red: toward the gradient; blue: away from the gradient). C) A Lifeact-RFP Jurkat transfectant migrating toward a $100 \mathrm{nM} \mathrm{SDF}-1 \alpha$ gradient.

\subsection{Internalization of CCR7}

CCR7 WT EGFP or CCR7 MT EGFP transfected Jurkat cells were stimulated with $100 \mathrm{nM}$ CCL19 or $100 \mathrm{nM}$ CCL21 for defined period of time. Cells were then quickly fixed with $4 \%$ paraformaldehyde (PFA) in PBS for $30 \mathrm{~min}$ at room temperature. Then cells were washed twice and stained with anti CCR7 Alexa647 antibody (BD Biosciences) for $30 \mathrm{~min}$ on ice. After washing twice, cells were analyzed by flow cytometry. The flow data were further analyzed using Flowjo. The mean fluorescence intensity (MFI) was measured and normalized to the control condition without chemokine stimulation to indicate the relative level of surface CCR7 expression.

\subsection{PDMS microfluidic device preparation and gradient generation}

The microfluidic devices used for cell migration experiments in this study were designed in Freehand 9.0 (Macromedia) and the design was printed to a transparency mask by a high resolution printer. The masters were fabricated in The Nano Systems Fabrication Laboratory (NSFL) at the University of Manitoba. The design was patterned on a silicon wafer by contact photolithography with SU 8 photoresist (Micro Chem, MA) through the transparency mask and the SU 8 pattern yields $\sim 100 \mu \mathrm{m}$ thickness The PDMS replicas were then fabricated by molding Polydimethylsiloxane (PDMS)(Sylgard 184 silicon elastomer, Dow Corning, MI) against the master. One millimeter 
A B
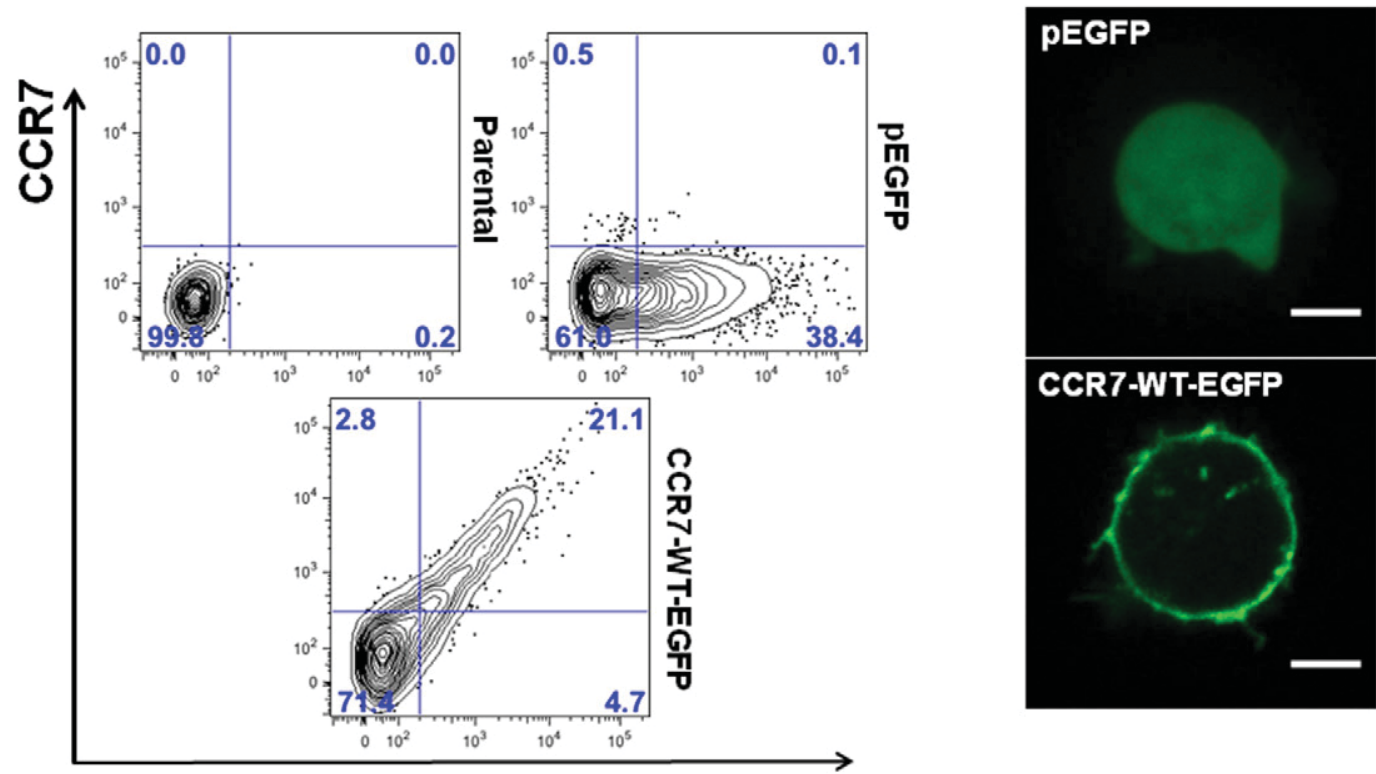

EGFP

C

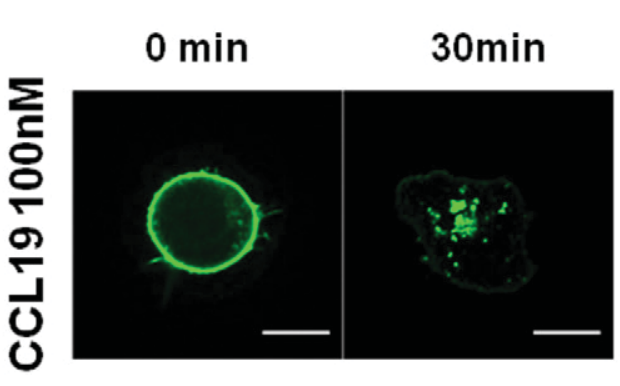

D

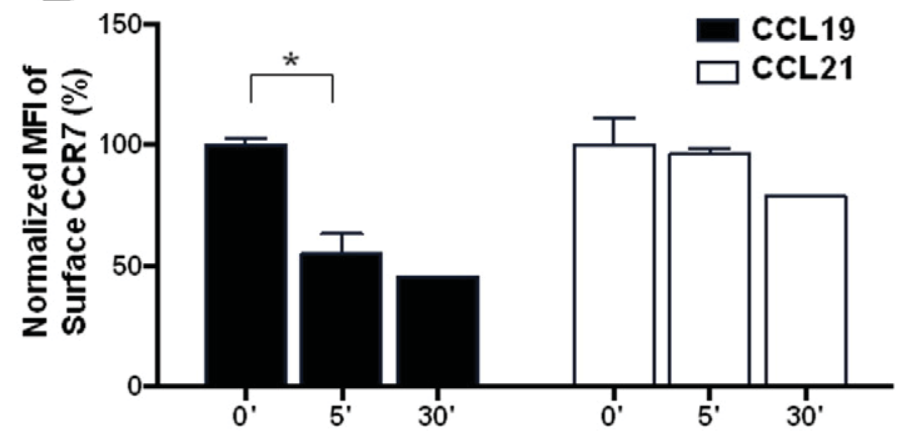

E

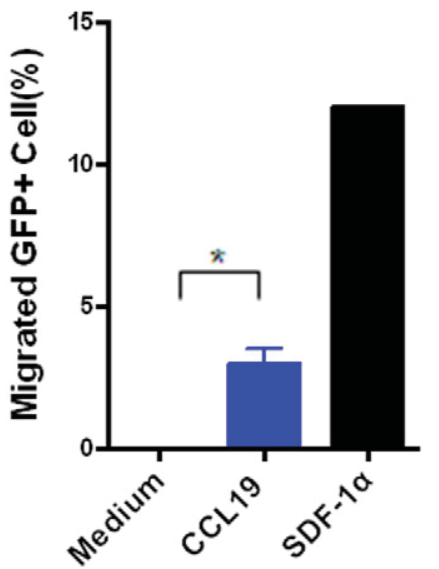


diameter holes for fluidic inlets and a $4 \mathrm{~mm}$ diameter hole for the fluidic outlet were punched out of PDMS respectively in each device. An additional $1 \mathrm{~mm}$ hole was punched for loading cells. Then the PDMS replica was bonded to a glass slide using an air plasma cleaner (PDC 32G, Harrick Plasma, NY). Polyethylene tubing (PE 20, Becton Dickinson, MD) was inserted into the inlet holes to connect the microfluidic device to syringe pumps (KDS 200, KD Scientific, MA) with two $1 \mathrm{~mL}$ BD TB syringes containing migration medium or chemokine solutions for fluidic infusion. Chemokine solutions (Recombinant Human CCL19/ MIP 3 beta and Recombinant Human CCL21/6Ckine from R\&D Systems; Recombinant Human SDF $1 \alpha /$ CXCL12 from Peprotech) of suitable concentrations were prepared in migration medium (RPMI 1640 with $0.4 \%$ BSA). The migra tion medium and chemokine solutions were continuously infused into the device by syringe pumps through tubing and the inlets of the device at the total flow rate of $0.4 \mu \mathrm{L} / \mathrm{min}$. The defined stable chemokine gradients are generated by controlled mixing of laminar flows of chemokines and medium. The mechanism for generating controlled stable chemical gradient in the "Y" device was characterized both experimentally and theoretically (Lin and Butcher, 2006). The cells were imaged $\sim 3 \mathrm{~mm}$ downstream of the "Y" junction.

\subsection{Microfluidic cell migration experiments and confocal microscopy}

The microfluidic cell migration experiment was per formed following the similar method described previously (Nandagopal et al., 2011). The fluidic channel was coated with fibronectin (BD Biosciences, MA) for $1 \mathrm{~h}$ at room temperature and blocked with BSA for another hour before the experiment. For each experiment, cells were loaded into the microfluidic device from the wells and allowed to settle in the fibronectin coated channel for $\sim 5 \mathrm{~min}$. The device was maintained at $37{ }^{\circ} \mathrm{C}$ and $5 \% \mathrm{CO}_{2}$ using a stage incubator (PECON, Germany). Transfectants migration was imaged using a $10 \times$ objective with a CSU X1M 5000 spinning disc confocal microscope (Carl Zeiss Canada). Cell migration in the applied gradient fields was recorded by time lapse microscopy at 1 frame/min for 15100 min using a CCD camera (Q32511, QIMAGING, Canada). A $63 \times$ objective was used to image CCR7 expression and internalization. A $488 \mathrm{~nm}$ solid state laser was used for imaging EGFP expression of the CCR7 transfectants and a $561 \mathrm{~nm}$ solid state laser was used for imaging RFP expression of the LifeAct transfectants. DIC images were taken in parallel. The image acquisition was controlled by ZEN2012 (Zeiss, Germany).

\subsection{Quantitative data analysis}

Movement of individual cells was tracked using $\mathrm{NIH}$ Image (v.1.47). The images were calibrated to distance. Only the cells that migrated within the microscope field throughout the time lapse period were selected and tracked using the "Manual Tracking" plug in in NIH ImageJ. The tracking data were exported to Excel. 32196 cell tracks in each individual experiment were analyzed. EGFP + cells were identified from the fluorescence images and then the corresponding cells were tracked using the DIC images for better tracking accuracy. At least 2 independent experiments were repeated for each condition with similar results. The figure used data from one representative experiment for each condition.

Following previously established analysis methods, the movement of cells was quantitatively evaluated by (1) the percentage of cells that migrated toward the chemokine gradient; (2) Chemotactic Index (C.I.), which is the ratio of the displacement of cells toward the chemokine gradient $(\Delta y)$, to the total migration distance $(d)$ using the equation C.I. $=\Delta y / d$, presented as the average value \pm standard error of the mean (SEM); (3) the average cell migration speed calculated as $d / \Delta t$ and presented as the average value \pm SEM of all cells. ibidi Chemotaxis tool (ibidi GmbH Munich), and MATLAB (The Math Works. Inc, MA) were used for data analysis.

\subsection{Statistical analysis}

Statistical analyses were performed using Graphpad Prism 6 (GraphPad Software. Inc, CA). Student's $t$ test was applied for statistical comparison of the data between different conditions $\left({ }^{*} p<0.05 ;{ }^{* *} p<0.01 ;{ }^{* * *} p<0.001 ;{ }^{* * * *} p<0.0001\right) . p<0.05$ is considered significantly different.

\section{Results}

\subsection{Migration of parental Jurkat cells and LifeAct RFP transfectants to a SDF $1 \alpha$ gradient}

Jurkat cells express CXCR4, a receptor for chemokine SDF $1 \alpha /$ CXCL12. Thus, we first used parental Jurkat cells to demonstrate the effective use of the microfluidic system for analyzing Jurkat cell migration and chemotaxis. Our results show that majority of the cells migrated toward a $100 \mathrm{nM}$ SDF $1 \alpha$ gradient in the microfluidic device [Fig. 1A B]. We also transiently transfected Jurkat cells with Lifeact RFP (an F actin binding probe) and tested the Lifeact RFP Jurkat transfectants migration in a $100 \mathrm{nM}$ SDF $1 \alpha$ gradient in the microfluidic device. We were able to observe individual Lifeact RFP Jurkat transfectant migrating toward the gradient [Fig. 1C]. The dynamic $\mathrm{F}$ actin organization in chemotaxing cell was visual ized by the Lifeact RFP signal [Fig. 1C; Video S1].

\subsection{Generation and characterizations of CCR7 Jurkat transfectants}

Our Jurkat cells express CCR7 at negligible low level (Fig. 2A). Therefore, these cells can be used as a parental T cell line for expressing transfected CCR7. We generated Jurkat

Fig. 2. Characterizations of CCR7 expression and dynamics in Jurkat transfectants. A) Cell surface CCR7 expression in parental Jurkat cells and Jurkat transfectants expressing transfected pEGFP or CCR7-WT-EGFP ( $48 \mathrm{~h}$ post transfection) measured by flow cytometry. B) Confocal images of Jurkat transfectants expressing pEGFP or CCR7-WT-EGFP. The scale bar indicates $10 \mu \mathrm{m}$. C) Confocal images of a representative Jurkat transfectant expressing CCR7-WT-EGFP upon stimulation of 100 nM CCL19 for $0 \mathrm{~min}$ or $30 \mathrm{~min}$. The scale bar indicates $10 \mu \mathrm{m}$. D) Internalization of CCR7 at different time points upon stimulation by $100 \mathrm{nM}$ CCL19 or CCL21 in CCR7-WT-EGFP Jurkat transfectants measured by CCR7 antibody staining and flow cytometry. E) Migration of CCR7-WT-EGFP Jurkat transfectants to medium control, 100 nM CCL19 or $100 \mathrm{nM}$ SDF- $1 \alpha$ using transwell assays. 
transfectants expressing transfected EGFP tagged wild type CCR7 (CCR7 WT EGFP). CCR7 WT EGFP showed significant correlation between EGFP and CCR7 [Fig. 2A]. The membrane bound CCR7 WT EGFP expression in transfectants was con firmed by confocal microscopy [Fig. 2B]. Furthermore, surface CCR7 WT EGFP in the Jurkat transfectants can be functionally internalized upon cellular stimulation with its ligand CCL19 as shown by confocal microscopy and FACS [Fig. 2C D]. By contrast, CCL21, another ligand for CCR7, induces a much lower level of internalization [Fig. 2D], which is consistent with the literatures (Kohout et al., 2004; Otero et al., 2008). These results established that CCR7 WT EGFP expressed in Jurkat cells is membrane associated and differentially responds to CCL19 and CCL21 ligands.

\subsection{Migration of CCR7 WT EGFP transfectants to CCL19 gradients}

In traditional transwell assays, the migration rate of CCR7 WT EGFP Jurkat transfectants to CCL19 is generally very low although it is significantly higher than the medium control [Fig. 2E]. The migration rate of the CCR7 WT EGFP Jurkat transfectants to SDF $1 \alpha$ mediated by endogenously expressed CXCR4 is higher [Fig. 2E]. Therefore, we believe the microfluidic based single cell migration analysis method is advantageous for studying lymphocyte transfectant migration. Using the microfluidic platform, we tested the migration of CCR7 WT EGFP Jurkat transfectants in two different CCL19 gradients, i.e. a single $10 \mathrm{nM}$ CCL19 gradient and a single $100 \mathrm{nM}$ CCL19 gradient. Our results show that $92.2 \%$ of the cells migrate toward the $100 \mathrm{nM}$ CCL19 gradient whereas only $60 \%$ of cells migrated toward the $10 \mathrm{nM}$ CCL19 gradient. This difference is confirmed by the cell migration trajectories and Chemotactic Index [Fig. 3A B; Video S2]. The cell migration speed is comparable in the $10 \mathrm{nM}$ and $100 \mathrm{nM}$ CCL19 gradients [Fig. 3B]. These results demonstrated the expected dose dependent chemotaxis of CCR7 Jurkat transfectants to CCL19 gradients.

\subsection{Migration of mutated CCR7 Jurkat transfectants to CCL19} gradients

We modified the previously characterized CCR7 MT2 mutant (the last 34 amino acids in the $C$ terminus were deleted)(Otero et al., 2008) by adding an in frame EGFP tag (for simplicity, we defined this construct as CCR7 MT EGFP in this study) [Fig. 4A]. CCL19 induced internalization of CCR7 MT EGFP was found to be reduced [Fig. 4B] consistent with the previous study using CCR7 MT2 pre B cell transfectants (Otero et al., 2008). When the $10 \mathrm{nM}$ CCL19 was applied in the microfluidic device, chemotaxis of CCR7 MT EGFP Jurkat transfectants was enhanced compared to that of the CCR7 WT EGFP transfectants [Fig. 4C D]. The cell migration speed is comparable between CCR7 WT EGFP and CCR7 MT EGFP Jurkat transfectants. These results demonstrate that the microfluidic device system is able to detect modified chemo tactic behavior determined by the chemokine gradient condi tion and CCR7 cytoplasmic tail.

\section{Discussion}

Genetic manipulations often affect cells' migration func tions. Electroporation based transfection methods disrupt cell

\section{A}

$10 \mathrm{nM}$

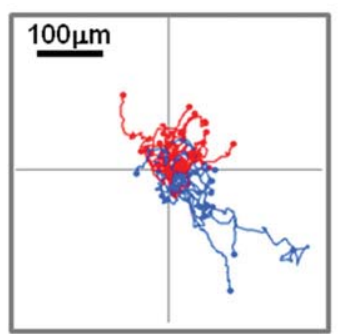

$100 \mathrm{nM}$

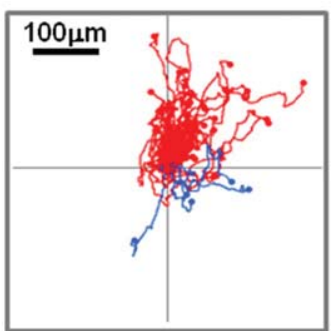

B

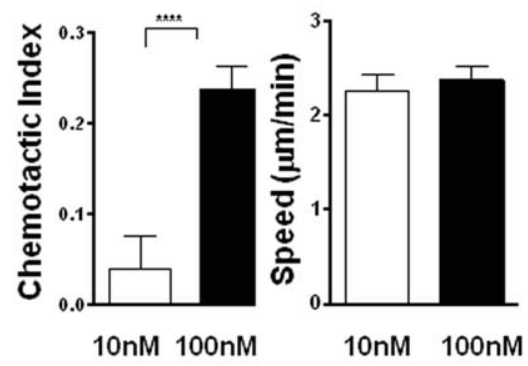

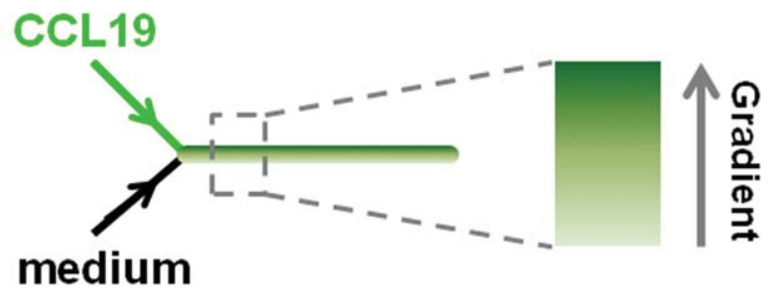

Fig. 3. Migration of CCR7-WT-EGFP Jurkat transfectants in CCL19 gradients using the microfluidic platform. A) Cell tracks of CCR7-WT-EGFP Jurkat transfectants with a common origin in a $10 \mathrm{nM}$ or a $100 \mathrm{nM}$ CCL19 gradient (red: toward the gradient; blue: away from the gradient) from a representative experiment. B) Quantitative analysis of Chemotactic Index (C.I.) and cell migration speed for the experiments in A). 

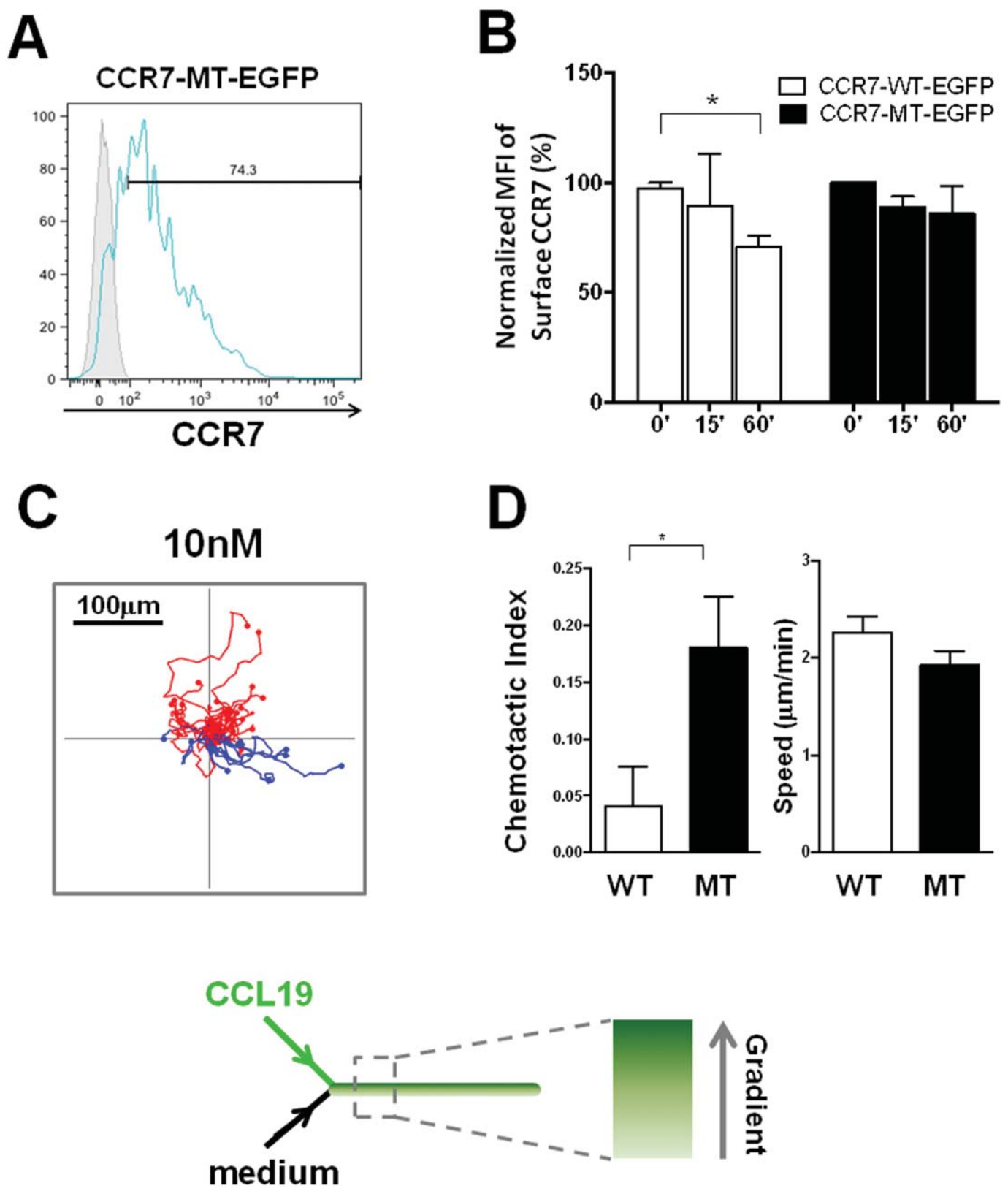

Fig. 4. Characterizations of CCR7-MT-EGFP expression and dynamics in Jurkat transfectants and migration of CCR7-MT-EGFP Jurkat transfectants to CCL19 gradients using the microfluidic platform. A) Surface expression of CCR7-MT-EGFP in Jurkat transfectants ( 48 h post transfection) measured by flow cytometry; B) comparison of CCL19 induced CCR7 internalization in CCR7-WT-EGFP and CCR7-MT-EGFP Jurkat transfectants at different time points measured by CCR7 antibody staining and flow cytometry. C) Cell tracks of CCR7-MT-EGFP Jurkat transfectants with a common origin in a $10 \mathrm{nM}$ CCL19 gradient (red: toward the gradient; blue: away from the gradient) from a representative experiment. D) Quantitative comparison of Chemotactic Index (C.I.) and cell migration speed between CCR7-MT-EGFP and CCR7-WTEGFP Jurkat transfectants in the $10 \mathrm{nM}$ CCL19 gradient.

membrane integrity (Chen et al., 2006; Kim and Eberwine, 2010), which can significantly influence the transfectants' adhesion and migration abilities. Compromise between trans fection efficiency and cell recovery after electroporation further complicates the use of the transfectants for migration studies. On the other hand, generation of stable transfectant lines is more time consuming and the transfectants can undergo genotypic and phenotypic changes over the clonal selection process. The complex cellular machinery operated by immune cells makes it less amenable to signaling interventions compar ing to other well established eukaryotic cell models such as Dictyostelium discoideum and yeast (Arkowitz, 1999; Iglesias, 2009; King and Insall, 2009; Lee and Jeon, 2012; Artemenko et al., 2014; Flor Parra et al., 2014). Our study addressed these challenges by optimizing the transfectant generation and microfluidic system. We successfully demonstrated the 
feasibility of studying the migration of transient CCR7 trans fectants in a relevant $\mathrm{T}$ cell line using a simple microfluidic gradient generator. Optimization of transfection efficiency and positive transfectants identification by EGFP tag makes this approach suitable for efficient cell migration analysis. We believe this developed method provides useful strategies for designing successful microfluidic cell migration studies with genetically manipulated $\mathrm{T}$ cell transfectants. It is worth pointing out that most experiments of this study were performed in an immu nology lab with an in house microfluidic device fabrication facility that demonstrates the feasibility of this approach.

In this study, we followed a previously reported strategy (Otero et al., 2008) to examine the $C$ terminus function of CCR7 for mediating $\mathrm{T}$ cell migration and chemotaxis. The specific CCR7 mutant with partial $\mathrm{C}$ terminus truncation has been shown to be capable of undergoing CCL19 induced internali zation in pre B 30019 transfectants (Otero et al., 2008). On the other hand, although not rigorously characterized, the mutant CCR7 mediates reduced CCR7 internalization in response to CCL19 stimulation comparing to wt CCR7 (Otero et al., 2008). In addition, the mutant CCR7 mediates similar chemotaxis of pre B 30019 transfectants comparing to wt CCR7 using transwell assays (Otero et al., 2008). In the present study, we also observed a general trend of reduction of CCL19 induced internalization of the mutant CCR7 in Jurkat transfectants comparing to wt CCR7. The exact time dependent dynamics of CCL19 induced CCR7 internalization can vary among different sets of experiments. Furthermore, we showed functional chemotaxis of mutant CCR7 transfectants in Jurkat cells to CCL19 gradients. In majority of the experiments to examine the migration of mutant CCR7 transfectants to a low dose CCL19 gradient, we observed an enhancement of chemotaxis, which is expected to result from the reduced CCR7 internalization. Further studies are required to draw concrete conclusions on the role of $\mathrm{C}$ terminus functions of CCR7 in mediating CCR7 signaling and $\mathrm{T}$ cell migration.

Supplementary data to this article can be found online at http://dx.doi.org/10.1016/j.jim.2015.02.008.

\section{Acknowledgments}

This study was supported by a Discovery Grant from the Natural Sciences and Engineering Research Council of Canada (NSERC) (RGPIN 201404789 and RGPIN 371474 2009) to F. Lin. F. Lin thanks the Winnipeg Rh Institute Foundation and the University of Manitoba for a Rh Award; X. Wu thanks the Faculty of Medicine and the Faculty of Graduate Studies at the University of Manitoba for travel awards and a fellowship. J.D. Wu thanks Research Manitoba for graduate fellowships. D.F. Legler is supported by the Swiss National Science Foundation (SNF31003A_143841). We thank Sen Hou and Christine Zhang for excellent research support. We thank the Nano Systems Fabrication Laboratory at the University of Manitoba for technical support.

\section{References}

Arai, Y., Shibata, T., Matsuoka, S., Sato, M.J., Yanagida, T., Ueda, M., 2010. Selforganization of the phosphatidylinositol lipids signaling system for random cell migration. Proc. Natl. Acad. Sci. 107, 12399.

Arkowitz, R.A., 1999. Responding to attraction: chemotaxis and chemotropism in Dictyostelium and yeast. Trends Cell Biol. 9, 20
Artemenko, Y., Lampert, T.J., Devreotes, P.N., 2014. Moving towards a paradigm: common mechanisms of chemotactic signaling in Dictyostelium and mammalian leukocytes. Cell. Mol. Life Sci. 71, 3711.

Ballestrem, C., Wehrle-Haller, B., Imhof, B.A., 1998. Actin dynamics in living mammalian cells. J. Cell Sci. 111 (Pt 12), 1649.

Bardi, G., Lipp, M., Baggiolini, M., Loetscher, P., 2001. The T cell chemokine receptor CCR7 is internalized on stimulation with ELC, but not with SLC. Eur. J. Immunol. 31, 3291.

Blow, N., 2007. Cell migration: our protruding knowledge. Nat. Methods 4, 589.

Bromley, S.K., Mempel, T.R., Luster, A.D., 2008. Orchestrating the orchestrators: chemokines in control of T cell traffic. Nat. Immunol. 9, 970.

Cahalan, M.D., Parker, I., 2008. Choreography of cell motility and interaction dynamics imaged by two-photon microscopy in lymphoid organs. Annu. Rev. Immunol. 26, 585

Campbell, J.J., Butcher, E.C., 2000. Chemokines in tissue-specific and microenvironment-specific lymphocyte homing. Curr. Opin. Immunol. 12, 336.

Cavnar, P.J., Berthier, E., Beebe, D.J., Huttenlocher, A., 2011. Hax1 regulates neutrophil adhesion and motility through RhoA. J. Cell Biol. 193, 465.

Chen, C., Smye, S.W., Robinson, M.P., Evans, J.A., 2006. Membrane electroporation theories: a review. Med. Biol. Eng. Comput. 44, 5

Deng, Q., Yoo, Sa K., Cavnar, Peter J., Green, Julie M., Huttenlocher, A., 2011. Dua roles for Rac2 in neutrophil motility and active retention in zebrafish hematopoietic tissue. Dev. Cell 21, 735.

Flor-Parra, I., Bernal, M., Zhurinsky, J., Daga, R.R., 2014. Cell migration and division in amoeboid-like fission yeast. Biol. Open 3, 108.

Forster, R., Schubel, A., Breitfeld, D., Kremmer, E., Renner-Muller, I., Wolf, E. Lipp, M., 1999. CCR7 coordinates the primary immune response by establishing functional microenvironments in secondary lymphoid organs. Cell 99, 23.

Forster, R., Davalos-Misslitz, A.C., Rot, A., 2008. CCR7 and its ligands: balancing immunity and tolerance. Nat. Rev. Immunol. 8, 362.

Fritschy, J.-M., Härtig, W., 2001. Immunofluorescence. eLS. John Wiley \& Sons, Ltd.

Gambardella, L., Vermeren, S., 2013. Molecular players in neutrophil chemotaxis-focus on PI3K and small GTPases. J. Leukoc. Biol. 94, 603.

Garside, P., Ingulli, E., Merica, R.R., Johnson, J.G., Noelle, R.J., Jenkins, M.K., 1998. Visualization of specific B and T lymphocyte interactions in the lymph node. Science 281, 96.

Gunn, M.D., Kyuwa, S., Tam, C., Kakiuchi, T., Matsuzawa, A., Williams, L.T. Nakano, H., 1999. Mice lacking expression of secondary lymphoid organ chemokine have defects in lymphocyte homing and dendritic cell localization. J. Exp. Med. 189, 451.

Heuzé, M.L., Vargas, P., Chabaud, M., Le Berre, M., Liu, Y.-J., Collin, O., Solanes, P., Voituriez, R., Piel, M., Lennon-Duménil, A.-M., 2013. Migration of dendritic cells: physical principles, molecular mechanisms, and functional implications. Immunol. Rev. 256, 240

Iglesias, P.A., 2009. Spatial regulation of PI3K signaling during chemotaxis. Wiley Interdiscip. Rev. Syst. Biol. Med. 1, 247.

Jin, T., Xu, X., Hereld, D., 2008. Chemotaxis, chemokine receptors and human disease. Cytokine 44, 1

John, B., Harris, T.H., Tait, E.D., Wilson, E.H., Gregg, B., Ng, L.G., Mrass, P., Roos, D.S., Dzierszinski, F., Weninger, W., Hunter, C.A., 2009. Dynamic imaging of CD8(+) T cells and dendritic cells during infection with Toxoplasma gondii. PLoS Pathog. 5, e1000505.

Keenan, T.M., Folch, A., 2008. Biomolecular gradients in cell culture systems. Lab Chip 8, 34 .

Kim, T.K., Eberwine, J.H., 2010. Mammalian cell transfection: the present and the future. Anal. Bioanal. Chem. 397, 3173.

King, J.S., Insall, R.H., 2009. Chemotaxis: finding the way forward with Dictyostelium. Trends Cell Biol. 19, 523.

Kohout, T.A., Nicholas, S.L., Perry, S.J., Reinhart, G., Junger, S., Struthers, R.S. 2004. Differential desensitization, receptor phosphorylation, beta-arrestin recruitment, and ERK1/2 activation by the two endogenous ligands for the CC chemokine receptor 7. J. Biol. Chem. 279, 23214.

Kunkel, E.J., Butcher, E.C., 2002. Chemokines and the tissue-specific migration of lymphocytes. Immunity 16,1 .

Lee, M.R., Jeon, T.J., 2012. Cell migration: regulation of cytoskeleton by Rap1 in Dictyostelium discoideum. J. Microbiol. 50, 555.

Li, J., Lin, F., 2011. Microfluidic devices for studying chemotaxis and electrotaxis. Trends Cell Biol. 21, 489.

Li, H., Hou, S., Wu, X., Nandagopal, S., Lin, F., Kung, S., Marshall, A.J., 2013. The tandem PH domain-containing protein 2 (TAPP2) regulates chemokineinduced cytoskeletal reorganization and malignant B cell migration. PLoS One 8, e57809.

Lin, F., Butcher, E., 2006. T cell chemotaxis in a simple microfluidic device. Lab Chip 6, 1462.

Lin, F., Baldessari, F., Gyenge, C.C., Sato, T., Chambers, R.D., Santiago, J.G., Butcher E.C., 2008. Lymphocyte electrotaxis in vitro and in vivo. J. Immunol. 181, 2465. 
Link, A., Vogt, T.K., Favre, S., Britschgi, M.R., Acha-Orbea, H., Hinz, B., Cyster, J.G., Luther, S.A., 2007. Fibroblastic reticular cells in lymph nodes regulate the homeostasis of naive T cells. Nat. Immunol. 8, 1255.

Long, A., Mitchell, S., Kashanin, D., Williams, V., Prina Mello, A., Shvets, I. Kelleher, D., Volkov, Y., 2004. A multidisciplinary approach to the study of T cell migration. Ann. N. Y. Acad. Sci. 1028, 313.

Luther, S.A., Bidgol, A., Hargreaves, D.C., Schmidt, A., Xu, Y., Paniyadi, J., Matloubian, M., Cyster, J.G., 2002. Differing activities of homeostatic chemokines CCL19, CCL21, and CXCL12 in lymphocyte and dendritic cell recruitment and lymphoid neogenesis. J. Immunol. 169, 424.

Miller, M.J., Wei, S.H., Parker, I., Cahalan, M.D., 2002. Two-photon imaging of lymphocyte motility and antigen response in intact lymph node. Science 296, 1869.

Moser, B., Loetscher, P., 2001. Lymphocyte traffic control by chemokines. Nat. Immunol. 2, 123.

Nandagopal, S., Wu, D., Lin, F., 2011. Combinatorial guidance by CCR7 ligands for T lymphocytes migration in co-existing chemokine fields. PLoS One 6 e18183.

Ngo, V.N., Lucy Tang, H., Cyster, J.G., 1998. Epstein-Barr virus-induced molecule 1 ligand chemokine is expressed by dendritic cells in lymphoid tissues and strongly attracts naive T cells and activated B cells. J. Exp. Med. 188, 181.

Otero, C., Groettrup, M., Legler, D.F., 2006. Opposite fate of endocytosed CCR7 and its ligands: recycling versus degradation. J. Immunol. 177, 2314.
Otero, C., Eisele, P.S., Schaeuble, K., Groettrup, M., Legler, D.F., 2008. Distinct motifs in the chemokine receptor CCR7 regulate signal transduction, receptor trafficking and chemotaxis. J. Cell Sci. 121, 2759

Ridley, A.J., Schwartz, M.A., Burridge, K., Firtel, R.A., Ginsberg, M.H., Borisy, G. Parsons, J.T., Horwitz, A.R., 2003. Cell migration: integrating signals from front to back. Science 302, 1704.

Riedl, J., Crevenna, A.H., Kessenbrock, K., Yu, J.H., Neukirchen, D., Bista, M. Bradke, F., Jenne, D., Holak, T.A., Werb, Z., Sixt, M., Wedlich-Soldner, R., 2008. Lifeact: a versatile marker to visualize F-actin. Nat. Methods 5, 605.

Robinson, I., Ochsenkühn, M.A., Campbell, C.J., Giraud, G., Hossack, W.J., Arlt, J., Crain, J., 2010. Intracellular imaging of host-pathogen interactions using combined CARS and two-photon fluorescence microscopies. J. Biophotonics 3, 138 .

Sai, J., Walker, G., Wikswo, J., Richmond, A., 2006. The IL sequence in the LLKIL motif in CXCR2 is required for full ligand-induced activation of Erk, Akt, and chemotaxis in HL60 cells. J. Biol. Chem. 281, 35931.

Servant, G., Weiner, O.D., Herzmark, P., Balla, T., Sedat, J.W., Bourne, H.R., 2000. Polarization of chemoattractant receptor signaling during neutrophil chemotaxis. Science 287, 1037.

Wang, Y., Shyy, J.Y., Chien, S., 2008. Fluorescence proteins, live-cell imaging, and mechanobiology: seeing is believing. Annu. Rev. Biomed. Eng. 10, 1.

Wu, J., Wu, X., Lin, F., 2013. Recent developments in microfluidics-based chemotaxis studies. Lab Chip 13, 2484. 